\title{
Chemically synthesized histone H2A Lys 13 di-ubiquitination promotes binding of 53BP1 to nucleosomes
}

Cell Research (2018) 28:257-260. doi:10.1038/cr.2017.157; published online15 December 2017

\section{Dear Editor,}

p53-binding protein 1 (53BP1) is a critical regulator of cellular response to DNA double-strand breaks (DSBs) [1]. To accomplish its repair function, 53BP1 must be recruited to the chromatin surrounding DSB sites that carry $\mathrm{H} 4$ methylation at Lys 20 and $\mathrm{H} 2 \mathrm{~A}$ ubiquitination at Lys15 [2-5]. The structural basis of this recognition process was recently revealed by the complex structure of 53BP1 bound to a nucleosome core particle (NCP) containing Lys20-dimethylated H4 (H4K20me2) and Lys15-mono-ubiquitinated H2A (H2AK15monoUb) [6]. It is fascinating to note that ubiquitin ligase RNF168 ubiquitinated H2A not only on Lys15, but also on Lys 13 without selectivity, and H2A bearing the K15Q mutation was still poly-ubiquitinated at Lys13 in vivo $[2-4,7]$. This leads to two questions. First, is 53BP1 also a reader of H2A Lys13 ubiquitin mark? Second, is poly-ubiquitination redundant at the 53BP1 recruitment event?

In previous studies, 53BP1 was considered as a specific reader of $\mathrm{H} 2 \mathrm{AK} 15$ monoUb, but not $\mathrm{H} 2 \mathrm{AK} 13$ monoUb $[5,6]$. Here, using chemically defined nucleosomes, we present the first evidence that 53BP1 can also recognize the H2A Lys13 di-ubiquitin mark. We first developed a practicable strategy for the total chemical synthesis of mono-/di-ubiquitinated histones to prepare nucleosomes. Surprisingly, we found that a NCP containing either Lys13- or Lys15-di-ubiquitinated H2A was effectively recognized by 53BP1. Moreover, 53BP1 preferentially interacted with the distal ubiquitin rather than the proximal ubiquitin in the H2AK13diUb. Further studies revealed that both H4K20me2 and the nucleosomal acidic patch are essential for the interaction. Together, our study suggested that H2A Lys13-poly-ubiquitination could also recruit 53BP1 in response to DNA damage.

To decipher the role of $\mathrm{H} 2 \mathrm{~A}$ ubiquitination in 53BP1 recognition, it is critical to generate site-specifically ubiquitinated H2As. However, the in vitro RNF168based enzymatic reaction was unable to discriminate between the adjacent Lys sites [6], and generated mainly mono-ubiquitinated histones $[2,8]$. Therefore, enzymatic approaches might not be suited for producing homogenous poly-ubiquitinated H2A of definite linkage and length [4], whereas chemical methods (as described in Supplementary information, Data S1) could prepare ubiquitinated histones with molecular homogeneity [9]. However, there are no reports yet about synthetic methods for preparing di-ubiquitinated histones. Accordingly, our studies commenced with the total chemical synthesis of the ubiquitinated H2As.

Initially, we implemented chemical synthesis of H2AK13monoUb. Its sequence was divided into five segments, namely 1, 2, 3, 4 and 5 . These segments were assembled through a convergent strategy, and auxiliary-mediated ligation of peptide hydrazide was applied to achieve site-specific ubiquitination (Supplementary information, Figures S1-S3). First, the ligation between segments 1 and 2 was conducted to furnish ubiquitin hydrazide 6 . Subsequently, peptide 6 was ligated with segment 3 , followed by auxiliary removal, to give the branched peptide 7 . In parallel, the ligation of segments 4 and 5 produced peptide 8 . After the condensation of peptides 7 and 8 , and subsequent desulfurization, H2AK13 monoUb was obtained with a total isolated yield of $15.7 \%$ (Figure 1B and 1C). Since K27-linked poly-ubiquitination of $\mathrm{H} 2 \mathrm{~A}$ is essential for repair signaling [2-4], we next prepared H2A bearing K27-linked di-ubiquitination at Lys13 or Lys15 (H2AK13diUb and H2AK15di$\mathrm{Ub})$. Following the above protocols, we first synthesized truncated mono-ubiquitin-modified H2A 13 (Figure 1A). In parallel, branched segment 9 was directly synthesized by employing orthogonal protection during SPPS (Supplementary information, Figure S4). Subsequently, segments 1, 9 and 13 were assembled by using N-to-C sequential ligations (Figure 1A and Supplementary information, Figure S5). After desulfurization, in total $\sim 13 \mathrm{mg}$ of $\mathrm{H} 2 \mathrm{AK} 13 \mathrm{diUb}$ was obtained with an overall yield of $7.8 \%$ (Figure 1B and 1C). Following these procedures, we also prepared H2AK15monoUb and H2AK15diUb (Figure 1B and 1C; Supplementary information, Figure S6). The subsequent $\mathrm{CD}$ analysis indicated that the synthetic proteins were properly folded as recombinant $\mathrm{H} 2 \mathrm{~A}$ 


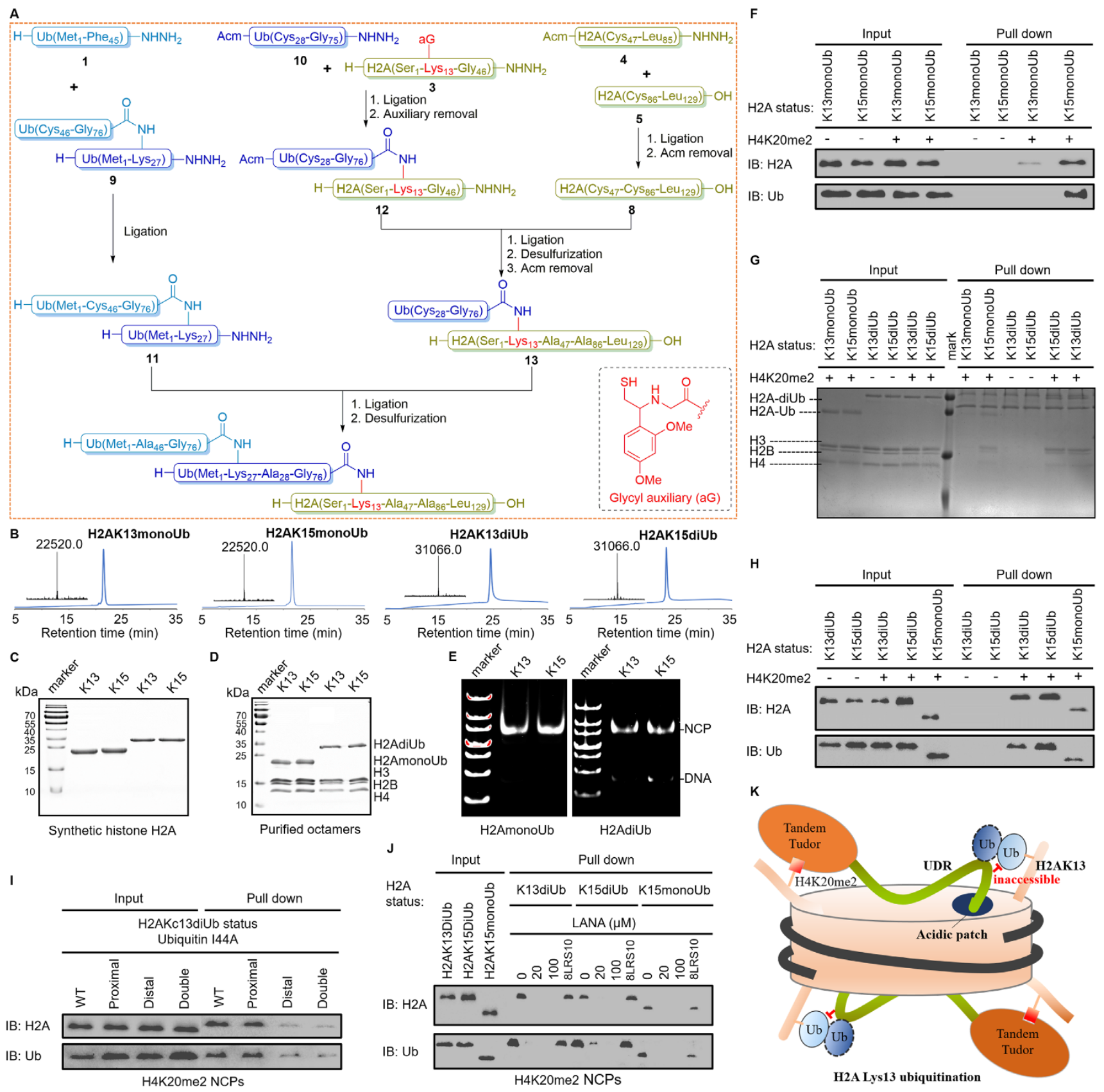

(Supplementary information, Figure S7). Next, the synthetic H2As were incorporated into histone octamers and nucleosomes with recombinant $\mathrm{H} 2 \mathrm{~B}, \mathrm{H} 3$, and chemically synthesized H4K20me2 (Figure 1D and 1E; Supplementary information, Figures S8, S12A-S12C).

With these nucleosomes in hands, we examined the interaction between 53BP1 and the modified NCPs. We performed pull-down experiments with GST-53BP1 fusion proteins (GST-Tudor-UDR, consisting of the tandem Tudor domain and ubiquitination-dependent recruitment region) as previously described [5]. We observed that 53BP1 selectively bound to NCPs containing H2AK15 monoUb, but not those containing H2AK13monoUb (Figure 1F). Moreover, specific interactions were only detected between 53BP1 and H4K20me2-containing NCPs (Figure 1F). These results were consistent with the previous studies $[5,6]$, verifying the bivalent binding of 53BP1 to NCPs containing both H4K20me2 and H2AK15 monoUb.

To investigate whether 53BP1 could selectively bind 
Figure 1 (A) Synthetic scheme for Lys13-di-ubiquitinated H2A. (B) RP-HPLC traces (214 nm) and deconvoluted ESI-MS (average isotope) spectra of purified H2As. H2AK13monoUb, observed: $22520.0 \mathrm{Da}$, calculated: $22523.1 \mathrm{Da}$; H2AK15monoUb, observed: 22520.0 Da, calculated: 22523.1 Da; H2AK13diUb, observed: 31066.0 Da, calculated: 31070.0 Da; H2AK15diUb, observed: 31066.0 $\mathrm{Da}$, calculated: $31070.0 \mathrm{Da}$. (C) SDS-PAGE analysis of the synthetic H2As, stained with Coomassie brilliant blue. (D) Reconstituted octamer samples containing H4K20me2 and ubiquitinated H2A were analyzed by SDS-PAGE. (E) Sybr Gold-stained native gels of reconstituted NCPs containing H4K20me2 and modified H2A. Mono-nucleosome and free 601 targeted sequence were marked as NCP and DNA, respectively. (F) GST-Tudor-UDR pull-down assays of NCPs containing mono-ubiquitinated H2A and H4K20me2 (+) or no H4K20me2 (-). IB, immunoblot. (G) Pull-down assays of NCPs containing di-ubiquitinated H2A and H4K20me2 (+) or no H4K20me2 (-), stained with Coomassie brilliant blue. H2AK13monoUb-containing NCPs with H4K20me2 were used as negative control; H2AK15monoUb-containing NCPs with H4K20me2 were used as positive control. (H) GST-Tudor-UDR pull-down assays of NCPs containing Lys13- or Lys15-di-ubiquitinated H2A and Lys20-methylated or -unmodified H4 and analysis by immunoblotting. $\mathrm{H} 2 \mathrm{AK} 15$ monoUb-containing NCPs with H4K20me2 were used as positive control. Modified H2As were detected by anti-H2A and anti-ubiquitin specific antibodies, respectively. (I) Pull-down assays of NCPs containing H4K20me2 and the indicated H2AKc13diUb mutants. The ubiquitin without or with I44A mutation was marked as WT, proximal, distal and double, respectively. (J) NCPs containing H4K20me2 and ubiquitinated H2As were pulled down by GST-Tudor-UDR, in the presence of LANA peptide or its mutant 8 LRS10 (100 $\mu \mathrm{M})$. LANA peptide of the indicated concentration $(0,20,100 \mu \mathrm{M})$ was added as a competitor in the pull-down assays. (K) Proposed model of 53BP1 binding to NCPs containing both H4K20me2 and H2AK13diUb. The effective binding involved intimate contacts with at least three elements, including the hydrophobic patch of ubiquitin, $\mathrm{H} 4 \mathrm{~K} 20 \mathrm{me} 2$ and the acidic patch. The distal ubiquitin contributed to the interaction, whereas the hydrophobic patch of proximal ubiquitin was inaccessible for $53 \mathrm{BP} 1$ binding.

to NCPs containing H2AK13diUb or H2AK15diUb, we incubated NCPs with GST-Tudor-UDR and conducted pull-down assays. To our surprise, SDS-PAGE analysis showed that 53BP1 indistinguishably bound to both $\mathrm{H} 2 \mathrm{AK} 13 \mathrm{diUb}$ - and H2AK15diUb-containing NCPs, as well as those containing $\mathrm{H} 2 \mathrm{AK} 15$ monoUb (Figure $1 \mathrm{G})$. However, H2AK13monoUb was not captured by GST-Tudor-UDR in the parallel test (Figure 1G). The pull-down experiments were repeated and analyzed by immunoblotting using anti-H2A and anti-ubiquitin specific antibodies, respectively. The results further confirmed that 53BP1 was not selective for $\mathrm{H} 2 \mathrm{AK} 13 \mathrm{diUb}$ or H2AK15diUb (Figure 1H). We next performed a competitive pull-down assay by mixing H2AK15monoUband $\mathrm{H} 2 \mathrm{AK} 13 \mathrm{diUb}-$ or H2AK15diUb-modified NCPs. We found that all the three ubiquitinated H2As were pulled down by GST-Tudor-UDR (Supplementary information, Figure S13). Moreover, the four ubiquitinated H2As except H2AK13monoUb could change the fluorescent lifetime of fluorescently labeled 53BP1 (Supplementary information, Figure S14). These results suggested that 53BP1 could not discriminate between the two closely positioned di-ubiquitinated Lys sites. Interestingly, when H2AK13diUb- or H2AK15diUb-containing NCPs without H4K20me2 was tested for binding to 53BP1, none of di-ubiquitinated histones were detected (Figure $1 \mathrm{G}$ and $1 \mathrm{H}$ ), indicating that $\mathrm{H} 4 \mathrm{~K} 20 \mathrm{me} 2$ was essential for the binding of 53BP1 to NCPs containing di-ubiquitinated $\mathrm{H} 2 \mathrm{~A}$. Together, these results suggested that 53BP1 might also be a bivalent binder of nucleosomes containing H2AK13diUb and H4K20me2.

Next, we tried to address the question of why 53BP1 bound to the NCPs containing H2AK13diUb, but not those containing H2AK13monoUb. Previous reports demonstrated that the hydrophobic patch centered at the Ile44 residue of ubiquitin contributed to the interaction between 53BP1 and H2AK15monoUb. The mutation of Ile44Ala (I44A) could dramatically impair their interaction $[5,6]$. However, the hydrophobic patch of H2AK13 monoUb was structurally inaccessible for 53BP1 binding [6]. Therefore, we hypothesized that H2AK13di$\mathrm{Ub}$ might provide an additional hydrophobic patch as a potential anchor for the binding. To verify this hypothesis, we repeated the binding experiments with a series of NCPs containing H2AK13diUb bearing I44A mutations in proximal and/or distal ubiquitin.

To facilitate examination of the interactions, we used a disulfide exchange method to prepare NCPs that contained Lys13-di-ubiquitinated H2A or its mutants [9], including $\mathrm{H} 2 \mathrm{AKc} 13 \mathrm{diUb}, \mathrm{H} 2 \mathrm{AKc} 13 \mathrm{diUb}^{\text {prox }}$ (proximal I44A mutation), H2AKc13diUb ${ }^{\text {dist }}$ (distal I44A mutation), H2AKc13diUb ${ }^{\text {doub }}$ (double I44A mutations) (Supplementary information, Figures S9-S11). Notably, the disulfide linkage did not interfere with the interaction, since $\mathrm{H} 2 \mathrm{AKc} 13 \mathrm{diUb}$ was captured by 53BP1 to a similar extent to natural H2AK13diUb (Supplementary information, Figure S15). When these NCPs were pulled down with GST-Tudor-UDR, we observed that H2AKc13di$\mathrm{Ub}^{\text {doub }}$-containing NCPs were barely captured by 53BP1. Interestingly, reduced 53BP1 binding with a similar extent was observed for $\mathrm{H} 2 \mathrm{AKc} 13 \mathrm{diUb}{ }^{\text {dist }}$. However, the I44A mutation in proximal ubiquitin did not affect the interaction (Figure 1I). These results indicated that the distal but not the proximal ubiquitin may dominate the binding of 53BP1 to H2AK13diUb-containing NCPs. However, the I44A mutation in either the proximal or distal ubiquitin slightly interfered with the interaction of 53BP1 with $\mathrm{H} 2 \mathrm{AKc} 15$ diUb, whereas double I44A muta- 
tions significantly impaired the binding (Supplementary information, Figure S16). This data indicated that either the proximal or distal ubiquitin of H2AKc15diUb may be accessible for 53BP1 binding.

The previous studies indicated a significant role of nucleosome acidic patch in 53BP1 binding, and demonstrated that the interaction between 53BP1 and $\mathrm{H} 2 \mathrm{AK}$ 15 monoUb-containing nucleosomes could be impaired by the acidic patch binding LANA peptide [6]. Therefore, we performed pull-down experiments in the presence or the absence of LANA peptide. As expected, the LANA peptide completely blocked the binding of 53BP1 to H2AK15monoUb-containing NCPs. The binding of 53BP1 with H2AK13diUb- or H2AK15diUb-containing NCPs was also outcompeted by the LANA peptide (Figure 1J). The mutant LANA(8LRS10) peptide, which has negligible binding to the acidic patch, was unable to block the interactions (Figure 1J). These data emphasized the significant role of the acidic patch for 53BP1 binding to $\mathrm{H} 2 \mathrm{AK} 13 \mathrm{diUb}$, consistent with previous reports of H2AK15monoUb [6].

Combining the above results and the previous structural information of 53BP1 bound to H2AK15monoUb-containing NCPs [6], we hypothesized a binding model in which intimate contacts between 53BP1 and di-ubiquitinated NCPs involve three elements, the hydrophobic patch, H4K20me2 and the nucleosome acidic patch (Figure $1 \mathrm{~K}$ and Supplementary information, Figure S17). The Lys13-tethered proximal ubiquitin was positioned in an off-conformation, whose hydrophobic patch was inaccessible for 53BP1 binding. However, the distal ubiquitin of $\mathrm{H} 2 \mathrm{AK} 13 \mathrm{diUb}$ could provide an additional hydrophobic patch to accommodate 53BP1 binding. Differentially, both the proximal and distal ubiquitins of H2AK15diUb were amenable to 53BP1 recognition. These strongly indicated the positional plasticity (further discussion in Supplementary information, Data S1) in recognition of H2A Lys13/15 ubiquitination. Besides, our results demonstrated the direct interaction between di-ubiquitinated $\mathrm{H} 2 \mathrm{~A}$ and 53BP1 in vitro. Nevertheless, the existence of $\mathrm{H} 2 \mathrm{~A}$ poly-ubiquitination on Lys 13 or Lys 15 still needs to be further demonstrated, since H2A Lys 15 mono-ubiquitination is adequate to recruit 53BP1 $[5,6]$.

In summary, we have established the first, to our knowledge, practicable strategy for the total chemical synthesis of di-ubiquitinated histones. This permitted us to constitute the first evidence that 53BP1 may also be a potential reader of H2A Lys13 poly-ubiquitination, which suggested the positional plasticity of $\mathrm{H} 2 \mathrm{~A}$ ubiquitination for 53BP1 recognition. The total chemical synthesis of di-ubiquitinated $\mathrm{H} 2 \mathrm{~A}$, together with the biochemical studies, pave the way for further structural and/or cell biology investigations into the roles of $\mathrm{H} 2 \mathrm{~A}$ ubiquitination in response to DNA damage.

Materials and Methods are available in Supplementary information, Data S2.

\section{Acknowledgments}

We thank Drs Rui-Ming Xu and Qing-Long You (Institute of Biophysics, Chinese Academy of Sciences) for kindly providing the GST-53BP1 plasmid. We also thank the Center for Biomedical Analysis of Tsinghua University for sample analysis. This work was supported by the National Key Research and Development Plan (2017YFA0505200 and 2016YFA0400903) and the National Natural Science Foundation of China (21532004, 81621002, 21621003 and 21708036).

\section{Jia-Bin $\mathrm{Li}^{1,{ }^{*}}$, Yun-Kun $\mathrm{Qi}^{2,3,{ }^{2}}$, Qiao-Qiao $\mathrm{He}^{2}$, Hua-Song $\mathrm{Ai}^{2}$, San-ling Liu ${ }^{1}$, Jia-Xing Wang ${ }^{2}$, Ji-Shen Zheng ${ }^{1}$, Lei Liu ${ }^{2}$, Changlin Tian ${ }^{1}$}

${ }^{I}$ Hefei National Laboratory for Physical Sciences at the Microscale and School of Life Sciences, University of Science and Technology of China, Hefei, Anhui 230027, China; ${ }^{2}$ Tsinghua-Peking Center for Life Sciences, Department of Chemistry, Tsinghua University, Beijing 100084, China; ${ }^{3}$ School of Pharmacy, Qingdao University, Qingdao, Shandong 266021, China

*These two authors contributed equally to this work.

Correspondence: Changlin Tian

E-mail: cltian@ustc.edu.cn

\section{References}

1 Panier R, Boulton SJ. Nat Rev Mol Cell Biol 2014; 15:7-18.

2 Mattiroli F, Vissers JHA, van Dijk WJ, et al. Cell 2012; 150:11821195.

3 Gatti M, Pinato S, Maspero E, et al. Cell Cycle 2012; 11:2538-2544.

4 Gatti M, Pinato S, Maiolica A, et al. Cell Rep 2015; 10:226-238.

5 Fradet-Turcotte A, Canny MD, Escribano-Diaz C, et al. Nature 2013; 499:50-54.

6 Wilson MD, Benlekbir S, Fradet-Turcotte A, et al. Nature 2016; 536:100-103.

7 Mattiroli F, Uckelmann M, Sahtoe DD, et al. Nat Commun 2014; 5:3291.

8 Hu Q, Botuyan MV, Cui G, et al. Mol Cell 2017; 66:473-487.

9 Fang GM, Li YM, Shen F, et al. Angew Chem Int Ed 2011; 50:76457649.

10 Chatterjee C, McGinty RK, Fierz B, et al. Nat Chem Biol 2010; 6:267269.

(Supplementary information is linked to the online version of the paper on the Cell Research website.) 